\title{
ON THE SYZYGIES AND HODGE THEORY OF NODAL HYPERSURFACES
}

\author{
ALEXANDRU DIMCA ${ }^{1}$
}

\begin{abstract}
We give sharp lower bounds for the degree of the syzygies involving the partial derivatives of a homogeneous polynomial defining an even dimensional nodal hypersurface. This implies the validity of formulas due to M. Saito, L. Wotzlaw and the author for the graded pieces with respect to the Hodge filtration of the top cohomology of the hypersurface complement in many new cases. A classical result by Severi on the position of the singularities of a nodal surface in $\mathbb{P}^{3}$ is improved and applications to deformation theory of nodal surfaces are given.
\end{abstract}

Dedicated to the memory of Alexandru Lascu, who was always searching for the Truth, and encouraging others to do the same.

\section{INTRODUCTION}

Let $S=\mathbb{C}\left[x_{0}, \ldots, x_{n}\right]$ be the graded ring of polynomials in $x_{0}, \ldots, x_{n}$ with complex coefficients and denote by $S_{r}$ the vector space of homogeneous polynomials in $S$ of degree $r$. For any polynomial $f \in S_{r}$, we define the Jacobian ideal $J_{f} \subset S$ as the ideal spanned by the partial derivatives $f_{0}, \ldots, f_{n}$ of $f$ with respect to $x_{0}, \ldots, x_{n}$ respectively, and we define the corresponding graded Milnor (or Jacobian) algebra by

$$
M(f)=S / J_{f}
$$

The Milnor algebra $M(f)$ can be seen (up to a twist in grading) as the top cohomology $H^{n+1}\left(K^{*}(f)\right)$, where $K^{*}(f)$ is the Koszul complex of $f_{0}, \ldots, f_{n}$ with the natural grading $\left|x_{j}\right|=\left|d x_{j}\right|=1$, defined by

$$
K^{*}(f): \quad 0 \rightarrow \Omega^{0} \rightarrow \Omega^{1} \rightarrow \ldots \rightarrow \Omega^{n+1} \rightarrow 0
$$

with all the arrows given by the wedge product by $d f=f_{0} d x_{0}+f_{1} d x_{1}+\ldots+f_{n} d x_{n}$. The homogeneous components of the next cohomology group, say $H^{n}\left(K^{*}(f)\right)_{n+r}$, describe the syzygies

$$
\sum_{j=0, n} a_{j} f_{j}=0
$$

where $a_{j} \in S_{r}$, modulo the trivial, or Koszul, syzygies generated by

$$
\left(f_{j}\right) f_{i}+\left(-f_{i}\right) f_{j}=0
$$

for all $i<j$. The following result was proved in [16].

2000 Mathematics Subject Classification. Primary 14C30, 13D40; Secondary 32S35, 13D02.

Key words and phrases. nodal hypersurfaces, syzygies, mixed Hodge structure, pole order filtration.

${ }^{1}$ Partially supported by Institut Universitaire de France. 
Theorem 1.1. Let $D: f=0$ be a nodal hypersurface of degree $d>2$ in $\mathbb{P}^{n}$.

(i) If $n=2 n_{1}+1$ is odd, then $H^{n}\left(K^{*}(f)\right)_{m}=0$ for any $m \leq n_{1} d$.

(ii) If $n=2 n_{1}$ is even, then $H^{n}\left(K^{*}(f)\right)_{m}=0$ for any $m \leq n_{1} d-1$.

Examples involving Chebyshev hypersurfaces shows that the bounds are best possible for $n$ even, but not for $n$ odd, see [16]. The first purpose of this paper is to establish the following new bound in the case $n$ odd, which is optimal, see Example 4.4 .

Theorem 1.2. Let $D: f=0$ be a nodal hypersurface of degree $d>2$ in $\mathbb{P}^{n}$.

If $n=2 n_{1}+1$ is odd, then $H^{n}\left(K^{*}(f)\right)_{m}=0$ for any $m \leq\left(n_{1}+1\right) d-\left[\frac{d}{2}\right]-1$.

A classical result of Severi [28] says that if a surface $D$ in $\mathbb{P}^{3}$ of degree $d$ has only nodes as singularities, then the set of nodes imposes independent conditions on hypersurfaces of degree $2 d-5$. A modern proof of this result can be found in [23], and a more general version is stated in [21, Corollary H]. Using Theorem 1.2 we can prove the following stronger version of Severi's result.

Corollary 1.3. Let $D: f=0$ be a nodal surface of degree $d>2$ in $\mathbb{P}^{3}$. Then the set of nodes imposes independent conditions on hypersurfaces of degree

$$
d+\left[\frac{d}{2}\right]-3 .
$$

The surfaces in $\mathbb{P}^{3}$ of degree $d$ are parametrized by $\mathbb{P}^{N}$, with $N=\left(\begin{array}{c}d+3 \\ 3\end{array}\right)-1$. There is a locally closed subscheme $V_{d, n} \subset \mathbb{P}^{N}$ parametrizing the surfaces $D \subset \mathbb{P}^{3}$ of degree $d$ and having exactly $n$ nodes, see [23], [29]. Let $X$ be the minimal resolution of a surface $D$ corresponding to a point in $V_{d, n}$ and let $R(X)$ be the formal moduli space of $X$ as in [3], regarded as a complete local ring. The first two claims of the following result are given in [23, Theorem 3.2] for the case $d=5$. The similar results in the case of nodal plane curves are classical, see [22] and [29, Corollary 4.7.8, Theorem 4.7.18 and Corollary 4.7.19].

Theorem 1.4. Fix a degree $d \in\{5,6,7\}$ and a positive integer $n$.

(1) The variety $V_{d, n}$ is a smooth locally closed subscheme of $\mathbb{P}^{N}$. If $V_{d, n} \neq \emptyset$, then $V_{d, n}$ has pure dimension $N-n$.

(2) If $V_{d, n} \neq \emptyset$, then $V_{d, n^{\prime}} \neq \emptyset$ for any positive integer $n^{\prime} \leq n$.

(3) The surface $X$ is unobstructed, i.e. the complete local ring $R(X)$ is regular, for any nodal surface $D \in V_{d, n}$.

In their paper [3], the authors give several examples of obstructed nodal surfaces for any degree $d \geq 8$. Our Theorem 1.4 explains why there are no such examples for $d<8$.

The idea of proof of Theorem 1.2 is similar to that used in the proof of Theorem 1.1, namely the interplay between Hodge filtrations, pole order filtrations and some spectral sequences. In the case of Theorem 1.1 it was enough to look at the cohomology of the complement $U=\mathbb{P}^{n} \backslash D$, while in the case at hand we have to consider 
the eigenspaces $H^{*}(F)_{\lambda}$ of the monodromy action on the corresponding Milnor fiber $F: f(x)-1=0$, which is technically more complicated.

The second aim of this paper is to show that the information we have obtained on the syzygies allows us to prove that an algebraic description of some of the graded pieces $G r_{F}^{p} H^{n}(U, \mathbb{C})$ of the top cohomology group $H^{n}(U, \mathbb{C})$ with respect to the Hodge filtration $F^{*}$ given in [14] holds in many cases, see Theorem 5.1, For basic facts on mixed Hodge structures we refer to the excellent monograph [24].

This paper was written back in 2013, and some of the results here were improved, both in generality and in presentation, in our subsequent joint work with Morihiko Saito, see [12], [13]. For instance, Theorem 1.2 is generalized to hypersurfaces with isolated weighted homogeneous singularities in [13, Theorem 9]. However, these preprints use a different approach and are not in the final form. Since our results have been already quoted in important papers such as [21], we have decided to publish them as an alternative view-point on this subject.

On the other hand, Corollary 1.3 and Theorem 1.4 above are new.

The author would like to thank Morihiko Saito and Edoardo Sernesi for very useful discussions related to this paper.

\section{Spectral Sequences for Milnor fibers of homogeneous polynomials}

For a fixed integer $k=1, \ldots, d$, we set $\lambda=\exp (-2 \pi i k / d)$ and let $L_{k}$ be the rank one local system on $U$ such that

$$
H^{*}\left(U, L_{k}\right)=H^{*}(F)_{\lambda},
$$

see for details [7, p. 211 and note that here the eigenspaces are with respect to the local system monodromy $T$ acting on $H^{*}(F)$, as explained in [11] .

Let $j: U \rightarrow X=\mathbb{P}^{n}$ be the inclusion, and let $\mathcal{L}_{k}$ be the meromorphic extension of $L_{k} \otimes_{\mathbb{C}} \mathcal{O}_{U}$. Then $\mathcal{L}_{k}$ is a regular holonomic $\mathcal{D}_{X}$-module, see [26], section (4.8), and one clearly has

$$
\mathbb{H}^{*}\left(X, D R\left(\mathcal{L}_{k}\right)\right)=\mathbb{H}^{*}\left(X, R j_{*} L_{k}\right)=H^{*}(F)_{\lambda} .
$$

The $\mathcal{D}_{X}$-module $\mathcal{L}_{k}$ has a natural (increasing) pole order filtration $P_{*}$ such that $P_{j} \mathcal{L}_{k}$ is isomorphic to $\mathcal{O}_{X}\left((j d+k)\right.$ for $j \in \mathbb{N}$ and $P_{j} \mathcal{L}_{k}=0$ for $j<0$, see (3.1.3) in [12]. We define a decreasing filtration $P^{*}$ on $\mathcal{L}_{k}$ by putting $P^{m}=P_{-m}$ for any $m \in \mathbb{Z}$. Then the de Rham complex $D R\left(\mathcal{L}_{k}\right)$ has an induced decreasing filtration $P^{*}$ and this induces the pole order filtration $P^{*}$ on the eigenspaces $H^{*}(F)_{\lambda}$ of the Milnor fiber cohomology. One has the following fundamental inclusion

$$
F^{s} H^{j}(F)_{\lambda} \subset P^{s} H^{j}(F)_{\lambda},
$$

for any $s$ and any $j$, where $F^{s}$ denotes the canonical Hodge filtration on the cohomology of the smooth quasi-projective variety $F$ constructed by P. Deligne, see (3.1.3), (4.4.7) and (4.4.8) in [12]. This extends the similar result for $H^{*}(U)$, see [5] and [25], which correspond to the case $\lambda=1$.

On the other hand, for each $k=1, \ldots, d$ there is a spectral sequence

$$
E_{1}^{p, q}(f, k)=H^{p+q}\left(K^{*}(f)\right)_{q d+k} \Rightarrow H^{p+q-1}(F)_{\lambda}
$$


coming from the graded Gauss-Manin complex $C_{f}^{*}$ associated with $f$, see (4.4.4) and (4.5.3) in [12. A similar spectral sequence is obtained by using the algebraic microlocal Gauss-Manin complex $\tilde{C}_{f}^{*}$ and the corresponding limit is the reduced cohomology of the Milnor fiber, see the (4.5) in [12]. In fact, one has

$$
H^{j+1}\left(C_{f}^{*}\right)_{k}=H^{j+1}\left(\tilde{C}_{f}^{*}\right)_{k}=H^{j}(F)_{\lambda}
$$

for any $k=1, \ldots, d$ and $j>0$, see (4.2.3) in [12]. These spectral sequences induce a filtration $P^{\prime}$ on $H^{*}(F)_{\lambda}$ and one has $P^{s}=P^{\prime s+1}$, see (4.4.7) in [12. When the hypersurface $D: f=0$ has only weighted homogeneous singularities, these spectral sequences degenerate at $E_{2}$, see the very recent paper by Morihiko Saito [27].

Remark 2.1. Remark 4.11 in [26] gives a very explicit description of the pole order filtration on the top cohomology group $H^{n}(F)$. Assume that we have a (finite) family of monomials $\left(g_{j}(x)\right)_{j \in J}$ in $S$ such that the cohomology classes $\left[\omega_{j}\right.$ ] of the differential forms $\omega_{j}=g_{j}(x) \Delta\left(d x_{0} \wedge \ldots \wedge d x_{n}\right)$ for $j \in J$ yield a basis of the $\mathbb{C}$-vector space $H^{n}(F)$. Then $G r_{P}^{n-q} H^{n}(F)_{\lambda}$ is spanned by the classes $\left[\omega_{j}\right]$ with $\operatorname{deg} \omega_{j}=q d+k$. Note that the eigenspace $G r_{P}^{n-q} H^{n}(F)_{1}$ is spanned exactly by the classes $\left[\omega_{j}\right]$ in $G r_{P}^{n-q} H^{n}(F)$ having a maximal degree $\operatorname{deg} \omega_{j}=(q+1) d$. In particular, the pole order filtrations on $H^{n}(F)_{1}$ and on $H^{n}(U)$, the latter constructed using a generalization of Griffiths approach in [6], Chapter 6 or in [17] are the same, as it should be since we have a natural isomorphism $H^{n}(F)_{1}=H^{n}(U)$. Note that the corresponding basis for $G r_{P}^{n-q} H^{n}(U)$ is usually written as

$$
\sigma_{j}=\omega_{j} / f^{q+1}
$$

Similarly, the limit term $E_{\infty}^{n-q+1, q}(f, k)$, which is isomorphic to $G r_{P}^{n-q} H^{n}(F)_{\lambda}$, has a basis given with the above notation by

$$
\eta_{j}=g_{j}(x) \cdot d x_{0} \wedge \ldots \wedge d x_{n} .
$$

\section{Hodge Filtration versus pole order FiLtration on Milnor Fibers}

If $D$ is a nodal hypersurface in $\mathbb{P}^{n}$, then one has an equality

$$
F^{s} H^{n}(U)=P^{s} H^{n}(U),
$$

for any $s \geq n-m+1$, with $m=\alpha_{D}=\frac{n}{2}$, see Corollary (0.12) in M. Saito [25], or the formula (1.1.3) in [14. The purpose of this section is to prove the following similar result for the associated Milnor fibers.

Proposition 3.1. Let $D: f=0$ be a nodal hypersurface of degree $d>2$ in $\mathbb{P}^{n}$ with $n$ odd. Then

$$
F^{s} H^{n}(F)_{\lambda}=P^{s} H^{n}(F)_{\lambda},
$$

for any $s \geq n-m+1$ and any $\lambda \in \mu_{d}, \lambda \neq 1$, with $m=\frac{n}{2}$.

Proof. Consider the hypersurface $\tilde{D}$ defined in $\mathbb{P}^{n+1}$ by the equation $\tilde{f}(x, t)=0$, with $\tilde{f}(x, t)=f(x)-t^{d}$. Let $\tilde{U}=\mathbb{P}^{n+1} \backslash \tilde{D}$ and let $H: t=0$ be the hyperplane at infinity in $\mathbb{P}^{n+1}$ such that $\tilde{U} \cap H=U$. Consider the Gysin long exact sequence

$$
\ldots \rightarrow H^{n-1}(U) \rightarrow H^{n+1}(\tilde{U}) \rightarrow H^{n+1}(\tilde{U} \backslash U) \rightarrow H^{n}(U) \rightarrow \ldots
$$


The group $\mu_{d}$ of $d$-roots of unity acts on $\mathbb{P}^{n+1}$ via

$$
\beta \cdot\left(x_{0}: \ldots: x_{n}: t\right)=\left(x_{0}: \ldots: x_{n}: \beta t\right) .
$$

This action extends the action of $\mu_{d}$ on $\mathbb{C}^{n+1}=\mathbb{P}^{n+1} \backslash H$, which is used to define the local system monodromy $T: F \rightarrow F$, namely

$$
\beta \cdot\left(x_{0}, \ldots, x_{n}\right)=\left(\beta^{-1} x_{0}, \ldots, \beta^{-1} x_{n}\right) .
$$

It follows that the Gysin exact sequence (3.2) inherits a $\mu_{d^{-}}$action, such that for any $\lambda \in \mu_{d}, \lambda \neq 1$, one has the following isomorphism of eigenspaces

$$
i^{*}: H^{n+1}(\tilde{U})_{\lambda} \rightarrow H^{n+1}(\tilde{U} \backslash U)_{\lambda} .
$$

On the other hand, one has $\tilde{U} \backslash U=\mathbb{C}^{n+1} \backslash F$, and hence a new Gysin sequence shows that one has an isomorphism

$$
R: H^{n+1}(\tilde{U} \backslash U)_{\lambda} \rightarrow H^{n}(F)_{\lambda},
$$

induced by a residue morphism $R$ which has Hodge type $(-1,-1)$. By composing the above two isomorphisms, we get isomorphisms

$$
R i^{*}: F^{s+1} H^{n+1}(\tilde{U})_{\lambda} \rightarrow F^{s} H^{n}(F)_{\lambda},
$$

for any integer $s$ and any $\lambda \in \mu_{d}, \lambda \neq 1$.

Now we look at the corresponding $P^{*}$ filtrations and show that

$$
\operatorname{dim} P^{s+1} H^{n+1}(\tilde{U})_{\lambda}=\operatorname{dim} P^{s} H^{n}(F)_{\lambda},
$$

for any integer $s$ and any $\lambda \in \mu_{d}, \lambda \neq 1$.

The cohomology of the filtered algebraic microlocal Gauss-Manin complexes $\tilde{C}_{f}^{*}$ and $\tilde{C}_{\tilde{f}}^{*}$ are closely related, namely

$$
H^{\ell+1}\left(\tilde{C}_{\tilde{f}}^{*}, P^{\prime}\right)=H^{\ell}\left(\tilde{C}_{f}^{*}, P^{\prime}\right) \otimes H^{1}\left(\tilde{C}_{t^{d}}^{*}, P^{\prime}\right)
$$

see (4.9) in [12]. Looking at the homogeneous components corresponding to $k=d$ and taking the $\lambda$-eigenspaces yields the equality (3.6) in view of (2.5).

Finally, any singularity of the hypersurface $\tilde{D}$ has type $A_{d-1}$, and hence the corresponding $\alpha_{\tilde{D}}$ is exactly $\tilde{m}=\frac{n}{2}+\frac{1}{d}$. Using Corollary (0.12) in M. Saito [25], or the formula (1.1.3) in [14, we see that

$$
F^{s} H^{n+1}(\tilde{U})=P^{s} H^{n+1}(\tilde{U}),
$$

for any $s \geq n-\tilde{m}+2$. Using the formulas (3.5) and (3.6) and the inclusion (2.3) we complete the proof, as one may clearly replace $\tilde{m}$ by $m$ as soon as $d>2$ and $n$ is odd.

Remark 3.2. Let $D: f=0$ be a curve in $\mathbb{P}^{2}$, with arbitrary isolated singularities. In this case an algorithm computing the dimension of the eigenspaces $H^{m}(F, \mathbb{C})_{\lambda}$, for $m=1,2$ and the dimension of the graded pieces of the pole order filtration on the Milnor fiber cohomology is described in [18]. Moreover, one has the following. 
(i) The Hodge filtration $F$ and the pole order filtration $P$ coincide on $H^{1}(F, \mathbb{C})_{\lambda}$ for any $\lambda$ in all the examples we have computed so far. We conjecture that the two filtrations $F$ and $P$ coincide on $H^{1}(F, \mathbb{C})_{\lambda}$ always.

(ii) On the other hand, the two filtrations $F$ and $P$ do not coincide on $H^{2}(F, \mathbb{C})_{\lambda}$ even in very simple cases, e.g. $C: f=\left(x^{2}-y^{2}\right)\left(x^{2}-z^{2}\right)\left(y^{2}-z^{2}\right)=0$ and $\lambda=-1$. A computation of the Hodge filtration on $H^{2}(F, \mathbb{C})$ in this case can be found in [1]. Note also that the mixed Hodge structure on $H^{2}(F, \mathbb{C})_{\neq 1}$ is not pure in general. For a line arrangement, one can use the formulas for the spectrum given in [2] to study the interplay between monodromy and Hodge filtration on $H^{2}(F, \mathbb{C})_{\neq 1}$.

\section{Proof of Theorem 1.2 And some examples}

Assume from now on that $n=2 n_{1}+1$. Then the equality of filtrations in Proposition 3.1 holds for $s \geq n_{1}+2$. We show now that this equality may be extended as follows.

Lemma 4.1. Let $D: f=0$ be a nodal hypersurface of degree $d>2$ in $\mathbb{P}^{2 n_{1}+1}$ and set $s=n_{1}+1$. Then

$$
F^{s} H^{n}(F)_{\lambda}=P^{s} H^{n}(F)_{\lambda}
$$

for $\lambda=\exp (-2 \pi i k / d)$ with

$$
0<k \leq k_{0}=d-\left[\frac{d}{2}\right]-1 .
$$

More precisely, in these conditions one has $\operatorname{dim} G r_{F}^{s} H^{n}(F)_{\lambda}=\operatorname{dim} G r_{P}^{s} H^{n}(F)_{\lambda}=$ $\operatorname{dim} M(f)_{n_{1} d+k-n-1}=\operatorname{dim} M(h)_{n_{1} d+k-n-1}$, where $h$ is a homogeneous polynomial in $S$ of degree d such that the hypersurface $D_{h}: h=0$ is smooth.

Proof. In view of the inclusions in (2.3) and using Proposition 3.1, it is enough to establish the inequality

$$
\operatorname{dim} G r_{F}^{s} H^{n}(F)_{\lambda} \geq \operatorname{dim} G r_{P}^{s} H^{n}(F)_{\lambda} .
$$

We know that

$$
\begin{gathered}
\operatorname{dim} G r_{P}^{s} H^{n}(F)_{\lambda}=\operatorname{dim} E_{\infty}^{n_{1}+2, n_{1}}(f, k) \leq \operatorname{dim} E_{1}^{n_{1}+2, n_{1}}(f, k)=\operatorname{dim} H^{n+1}\left(K^{*}(f)\right)_{n_{1} d+k}= \\
=\operatorname{dim} M(f)_{n_{1} d+k-n-1}=\operatorname{dim} M(h)_{n_{1} d+k-n-1}
\end{gathered}
$$

where $h \in S_{d}$ denotes a polynomial such that the associated hypersurface $D_{h}: h=0$ is smooth. Indeed, the last equality follows from Corollary 2.2. $(i)$ in [16], which is a direct consequence of Theorem 1.1.

On the other hand, using an argument similar to Proposition 4.1 in [8], which goes back to Lemma 3.6. in [26], it follows that for $\beta \neq \pm 1$, the mixed Hodge structure induced on

$$
H^{n}(F)_{\beta, \bar{\beta}}=H^{n}(F)_{\beta} \oplus H^{n}(F)_{\bar{\beta}}
$$

is pure of weight $n$. Therefore

$$
\operatorname{dim} G r_{F}^{s} H^{n}(F)_{\lambda}=h^{n_{1}+1, n_{1}}\left(H^{n}(F)_{\lambda}\right)=h^{n_{1}+1, n_{1}}\left(H^{n}(\tilde{D})_{\lambda}\right)
$$


in view of Corollary 1.2 in [15]. To compute the last equivariant Hodge number we use Proposition 5.2 in [15]. The first term in the sum giving $h^{n_{1}+1, n_{1}}\left(H^{n}(\tilde{D})_{\lambda}\right)$ is the corresponding number computed for the smooth hypersurface $\tilde{D}_{h}: \tilde{h}=h(x)-t^{d}=0$. Using the standard identification going back to Griffiths [20],

$$
H^{n_{1}+1, n_{1}}\left(H^{n}\left(\tilde{D}_{h}\right)\right)=H^{n+2}\left(K^{*}(\tilde{h})\right)_{\left(n_{1}+1\right) d},
$$

and recalling that taking the $\lambda$-eigenspace means to look at forms of the form

$$
g(x) t^{d-k-1} d x_{0} \wedge \ldots \wedge d x_{n} \wedge d t
$$

where $g(x)$ is homogeneous of degree $n_{1} d+k-n-1$, we get

$$
h^{n_{1}+1, n_{1}}\left(H^{n}\left(\tilde{D}_{h}\right)_{\lambda}\right)=\operatorname{dim} M(h)_{n_{1} d+k-n-1} .
$$

We show now that the other terms in the sum giving $h^{n_{1}+1, n_{1}}\left(H^{n}(\tilde{D})_{\lambda}\right)$ are trivial. These terms are of two types:

(a) $h^{p, q}\left(H^{n+1}(\tilde{D})_{\lambda}\right)$, which are zero since a $\beta$-eigenspace of the group $H^{n+1}(\tilde{D})$ under the $\mu_{d}$-action maybe nontrivial only for $\beta= \pm 1$, see Example 6.3.24 in [6], Theorem 1.1 in [15] and Theorem 4.1 in [16].

(b) $h^{p, q}\left(H^{n}(F(d))_{\lambda}\right)$, where $F(d)$ is the affine Milnor fiber given by

$$
g(y, t)=y_{1}^{2}+\ldots y_{n}^{2}+t^{d}-1=0
$$

in $\mathbb{C}^{n+1}$ with the corresponding $\mu_{d}$-action, i.e.

$$
\beta\left(y_{1}, \ldots, y_{n}, t\right)=\left(y_{1}, \ldots, y_{n}, \beta t\right)
$$

replacing the monodromy action when eigenspaces are considered. Since this is the Milnor fiber of an isolated weighted homogeneous singularity whose link is a rational homology sphere, we have $h^{p, q}\left(H^{n}(F(d))_{\lambda}\right)=0$ for $p+q \neq n$. It follows that we have just one such number to investigate, namely $h^{n_{1}+1, n_{1}}\left(H^{n}(F(d))_{\lambda}\right)$. Using the weights $w t\left(y_{j}\right)=2$ and $w t(t)=d$, we get using [32]

$$
h^{n_{1}+1, n_{1}}\left(H^{n}(F(d))=\sum_{j=1,2 d-1} \operatorname{dim} M(g(y, t))_{j-d-2} .\right.
$$

The $\lambda$-eigenspace should come from the monomial $t^{d-k-1}$, of degree $2 d-2 k-2$. Our condition on $k$ implies that $2 d-2 k-2>d-3$, hence this monomial is not giving a contribution to $h^{n_{1}+1, n_{1}}\left(H^{n}(F(d))\right.$, i.e. $h^{n_{1}+1, n_{1}}\left(H^{n}(F(d))_{\lambda}\right)=0$. Moreover $2 k<d$ in order to avoid the case $\lambda=-1$.

This shows that $\operatorname{dim} G r_{F}^{s} H^{n}(F)_{\lambda}=\operatorname{dim} M(h)_{n_{1} d+k-n-1}$, completing the proof of Lemma 4.1.

Now we give the proof of Theorem 1.2, Lemma 4.1 implies that

$$
E_{1}^{n_{1}+2, n_{1}}\left(f, k_{0}\right)=H^{n+1}\left(K^{*}(f)\right)_{n_{1} d+k_{0}}=E_{\infty}^{n_{1}+2, n_{1}}\left(f, k_{0}\right) .
$$

Moreover, $E_{1}^{n_{1}+2+e, n_{1}-e}\left(f, k_{0}\right)=E_{\infty}^{n_{1}+1+e, n_{1}-e}\left(f, k_{0}\right)$ for all $e=1,2, \ldots, n_{1}$ by similar (and simpler) computations based on Proposition 3.1. It follows that all the differentials in the spectral sequence $E_{r}\left(f, k_{0}\right)$ starting from $E_{r}^{n_{1}+1, n_{1}}\left(f, k_{0}\right)$ are 0 . Since 
$E_{\infty}^{n_{1}+1, n_{1}}\left(f, k_{0}\right)=0$ as well (the only eigenvalues of the monodromy on $H^{n-1}(F)$ are \pm 1 ), we get

$$
E_{1}^{n_{1}+1, n_{1}}\left(f, k_{0}\right)=H^{n}\left(K^{*}(f)\right)_{n_{1} d+k_{0}}=0
$$

which completes the proof of Theorem 1.2. Indeed, recall that if the coordinates $x_{0}, \ldots, x_{n}$ are choosen such that the hyperplane at infinity $H_{0}: x_{0}=0$ is transversal to $D$, then the multiplication by $x_{0}$ induces an injection $H^{n}\left(K^{*}(f)\right)_{s-1} \rightarrow H^{n}\left(K^{*}(f)\right)_{s}$ for any $s$ (the dual statement for the homology is part of Corollary 11 in [4]).

Before proceeding, we recall the following notions, introduced in [17].

Definition 4.2. For a hypersurface $D: f=0$ of degree $d$ with isolated singularities we introduce three integers, as follows:

(i) the coincidence threshold ct $(D)$ defined as

$$
c t(D)=\max \left\{q: \operatorname{dim} M(f)_{k}=\operatorname{dim} M(h)_{k} \text { for all } k \leq q\right\},
$$

with $h$ a homogeneous polynomial in $S$ of degree $d=\operatorname{deg} f$ such that $D_{h}: h=0$ is a smooth hypersurface in $\mathbb{P}^{n}$.

(ii) the minimal degree of a nontrivial syzygy $m d r(D)$ defined as

$$
m d r(D)=\min \left\{q: H^{n}\left(K^{*}(f)\right)_{q+n} \neq 0\right\}
$$

where $K^{*}(f)$ is the Koszul complex of $f_{0}, \ldots, f_{n}$ with the natural grading defined in [17].

Moreover it is easy to see that one has

$$
c t(D)=\operatorname{mdr}(D)+d-2 .
$$

In practice, for a given polynomial $f$, it is easy to compute $c t(D)$ using a number of computer algebra softwares.

Corollary 4.3. Let $D: f=0$ be a nodal hypersurface of degree $d>2$ in $\mathbb{P}^{n}$. If $n=2 n_{1}+1$ is odd, then

$$
\operatorname{ct}(D) \geq\left(n_{1}+2\right) d-\left[\frac{d}{2}\right]-n-2 .
$$

Example 4.4. Let $\mathcal{C}(3, d)$ be the Chebyshev surface of degree $d$ in $\mathbb{P}^{3}$ as defined in [16]. Then for $3 \leq d \leq 20$, numerical computation shows that one has

$$
\operatorname{ct}(\mathcal{C}(3, d))=3 d-\left[\frac{d}{2}\right]-5,
$$

i.e. we have equalities for these cases in Corollary 4.3, It follows that in any such case the bound for the vanishing in Theorem 1.2 is sharp, namely

$$
H^{n}\left(K^{*}(f)\right)_{m+1} \neq 0
$$

for $m=\left(n_{1}+1\right) d-\left[\frac{d}{2}\right]-1$. 
Example 4.5. Let $D$ be a nodal hypersurface of degree $d$ in $\mathbb{P}^{n}$ having exactly one singularity. Then it is known that

$$
m d r(f)=n(d-2) \text { and } c t(D)=(n+1)(d-2),
$$

see [17], i.e. a substantially bigger number that the lower bound given in Corollary 4.3. A better lower bound for $\operatorname{ct}(D)$ than that given by Corollary 4.3 in the case of a nodal hypersurface with not too many nodes is given in [10]. More precisely, it is shown that

$$
m d r(f) \geq n(d-2)+1-\sharp A_{1} \text { and } c t(D) \geq(n+1)(d-2)+1-\sharp A_{1},
$$

where $\sharp A_{1}$ denotes the number of nodes of the nodal hypersurface $D$. Note that these inequalities are sharp when $\sharp A_{1}=1$. For the case of several nodes which are linearly independent, see [12, Proposition 1].

Remark 4.6. Let $D: f=0$ be a degree $d$ hypersurface in $\mathbb{P}^{n}$ having only isolated singularities. Let $\hat{J}$ be the saturation of the Jacobian ideal $J$ of $f$. Then the vector space $\hat{J}_{d} / J_{d}$ is naturally identified with the space of first order locally trivial deformations of $D$ in $\mathbb{P}^{n}$ modulo those arising from the above $P G L(n+1)$-action, see E. Sernesi [31]. The dimension of the vector space $\hat{J}_{d} / J_{d}$ can be determined as follows.

$$
\operatorname{dim} \hat{J}_{d} / J_{d}=\operatorname{dim} M(f)_{d}-\operatorname{dim} M(h)_{d}+\operatorname{dim} M(f)_{T-d}-\tau(D),
$$

where $h$ is as in Definition [4.2, see G. Sticlaru [33] for this formula and a number of interesting examples of rigid and non-rigid hypersurfaces. Here $\tau(D)$ is the total Tjurina number of the hypersurface $D$, e.g. the number of nodes for a nodal hypersurface.

\section{Hodge theory of NODAL HyPERSuRFACES AND THE PROOF OF Corollary 1.3 AND TheOREM 1.4}

Let $\mathcal{I} \subset \mathcal{O}_{\mathbb{P}^{n}}$ be the reduced ideal sheaf of the set of nodes $\mathcal{N}=\operatorname{Sing} D \subset \mathbb{P}^{n}$. Set $I_{k}(i)=H^{0}\left(\mathbb{P}^{n}, \mathcal{I}^{i}(k)\right) \subset S_{k}$ and define $I^{(i)}=\oplus_{k} I_{k}(i)$, a homogeneous ideal in the polynomial ring $S$.

For a degree $d$ nodal hypersurface $D$ in $\mathbb{P}^{n}$, one of the main results in [14] describe the graded pieces $G r_{F}^{p} H^{n}(U)$ of the top cohomology of the complement $U=\mathbb{P}^{n} \backslash D$ with respect to the Hodge filtration $F$ in terms of purely algebraic objects, namely one has

$$
G r_{F}^{p} H^{n}(U, \mathbb{C})=\left(I^{(q-m+1)} / I^{(q-m)} J_{f}\right)_{(q+1) d-n-1}
$$

for $q=n-p>m:=\left[\frac{n}{2}\right]$ under a certain condition (B), see Theorem 2 in [14].

Recall that for a finite set of points $\mathcal{N} \subset \mathbb{P}^{n}$ we denote by

$$
\operatorname{def} S_{m}(\mathcal{N})=|\mathcal{N}|-\operatorname{codim}\left\{h \in S_{m} \mid h(a)=0 \text { for any } a \in \mathcal{N}\right\},
$$

the defect (or superabundance) of the linear system of polynomials in $S_{m}$ vanishing at the points in $\mathcal{N}$, see [6], p. 207. This positive integer is called the failure of $\mathcal{N}$ to impose independent conditions on homogeneous polynomials of degree $m$ in [19]. There is a close relationship between defects $\operatorname{def} S_{m}(\mathcal{N})$ and the syzygies described by $H^{n}\left(K^{*}(f)\right)$, see [17] for nodal hypersurfaces and [9] for projective hypersurfaces 
with isolated singularities. More precisely, for the nodal hypersurfaces one has the following.

$$
\operatorname{def} S_{k}(\mathcal{N})=\operatorname{dim} H^{n}\left(K^{*}(f)\right)_{n d-n-1-k},
$$

for $0 \leq k \leq n d-2 n-1$ and $\operatorname{def} S_{k}(\mathcal{N})=0$ for $k>n d-2 n-1$.

The discussion following the statement of Theorem 2 in [14] shows that in fact (B) is equivalent to the condition

$$
\left(B^{\prime}\right): \operatorname{def} S_{e}(\mathcal{N})=0,
$$

where $e=\left[\frac{n}{2}\right](d-1)-p$ and $\mathcal{N}$ is the set of nodes of $D$.

One has the following consequence of Theorems 1.1 and 1.2 .

Theorem 5.1. Let $D: f=0$ be a nodal hypersurface of degree $d>2$ in $\mathbb{P}^{n}$ and assume that $q=n-p>m:=\left[\frac{n}{2}\right]$.

(i) If $n=2 n_{1}$ is even, then the isomorphism (5.1) always hold.

(ii) If $n=2 n_{1}+1$ is even, then the isomorphism (5.1) holds if either

$$
p \leq n-\left[\frac{n}{2}\right]-\left[\frac{d}{2}\right]
$$

or

$$
\sharp A_{1} \leq\left(n_{1}+2\right)(d-1)+p-n .
$$

In particular, the isomorphism (5.1) holds always for $d=3$, and for $d=4$ with the additional condition $\sharp A_{1} \leq n_{1}+p+5$.

Proof. When $n=2 n_{1}$ is even, then $p<n_{1}$ and hence $e=n_{1}(d-1)-p=n_{1} d-n_{1}-$ $p>n_{1} d-n$. Use now Corollary 2.2 (ii) in [16] which says that $\operatorname{def} S_{k}(\mathcal{N})=0$ for $k \geq n_{1} d-n$.

When $n=2 n_{1}+1$, we have $e=n_{1}(d-1)-p$. On the other hand, one know that $\operatorname{def} S_{k}(\mathcal{N})=0$ if and only if $k \geq T-c t(D)$, see Theorem 1.5 in [17]. Corollary 4.3 implies

$$
T-\operatorname{ct}(D) \leq 2\left(n_{1}+1\right)(d-2)-\left(\left(n_{1}+2\right) d-\left[\frac{d}{2}\right]-n-2\right)=n_{1} d-n+\left[\frac{d}{2}\right] .
$$

Hence $e \geq n_{1} d-n+\left[\frac{d}{2}\right]$ as soon as $p \leq n-\left[\frac{n}{2}\right]-\left[\frac{d}{2}\right]$. For the claims involving $\sharp A_{1}$, use Example 4.5.

Note that Example 4.7 in [14] shows that (B) may not be satisfied by a nodal surface, where $n=3, d=4, p=1$ and $\sharp A_{1}$ is large. This shows that the first condition in Theorem 5.1 (ii) is sharp, as in the case at hand we get $1 \leq 3-1-2$, which fails just by 1 . The following result generalizes Corollary 1 in [14].

Corollary 5.2. Let $D: f=0$ be a nodal hypersurface of degree $d \geq 5$ in $\mathbb{P}^{n}$. If $n$ is odd and $\sharp A_{1} \leq n+p+6$, then the isomorphism (5.1) holds. 
Now we give the proof of Corollary 1.3 . When $n=3$, then by Theorem 1.2 we get $H^{3}\left(K^{*}(f)\right)_{m}=0$ for any $m \leq 2 d-\left[\frac{d}{2}\right]-1$. Using formula (5.2), this implies $\operatorname{def} S_{k}(\mathcal{N})=0$ for $k \geq d+\left[\frac{d}{2}\right]-3$, which is exactly the claim in Corollary 1.3. Note that for $d>2$ we have

$$
d+\left[\frac{d}{2}\right]-3 \leq 2 d-5
$$

with equality only for $d=3$ and $d=4$. In other words, our result is stronger than Severi's for any $d \geq 5$.

The proof of the first two claims in Theorem 1.4 follows from Corollary 1.3 and the discussion in [23, (3.6)]. Indeed, one can check that the inequality

$$
d+\left[\frac{d}{2}\right]-3 \leq d
$$

holds exactly for $d \leq 7$. To prove the third claim, note that one has

$$
\operatorname{dim} m_{R(X)} / m_{R(X)}^{2}-\operatorname{dim} R(X)=\operatorname{def} S_{d}(\mathcal{N}),
$$

for $d \geq 5$, see [3, Theorem 4.2], where $m_{R(X)}$ denotes the maximal ideal of the complete local ring $R(X)$.

Remark 5.3. (1) Note that the Krull dimension $\operatorname{dim} R(X)$ of the formal moduli space $R(X)$ of $X$ is given by $N-15$, see [3, Corollary 2.11], which is in general different from $\operatorname{dim} V_{d, n}$. In other words, the (embedded) deformation theory of the nodal surface $D$, reflected in the germ of $V_{d, n}$ at $D$, is quite different from the deformation theory of the surface $X$, reflected in the local $\operatorname{ring} R(X)$.

(2) For a surface $D$ in $\mathbb{P}^{3}$ of degree $d \leq 4$ with only rational double points, one has that the corresponding minimal surface $X$ is unobstructed, see $[3$, Example (4.7)]. In the same Example, the authors produce a quintic $D$ with $10 A_{4}$-singularities which is obstructed.

(3) It is very likely that the second claim in Theorem 1.4 can be upgraded to the inclusion $V_{d, n} \subset \bar{V}_{d, n^{\prime}}$, as it is the case for the Severi varieties of nodal plane curves, see [22] or [29, Theorem 4.7.18]. To do this, one should check that the methods described in [22] or [30] work in the case of nodal surfaces as well.

\section{REFERENCES}

[1] P. Bailet, Milnor fibers of hyperplane arrangements, PhD Thesis, Nice 2014. 3.2

[2] N. Budur, M. Saito, Jumping coefficients and spectrum of a hyperplane arrangement, Math. Ann. 347 (2010), 545-579. 3.2

[3] D.M. Burns, J. M. Wahl, Local contributions to global deformations of surfaces, Invent. Math. 26(1974), 67-88. 1, 1] 5, 1] 2]

[4] A. D. R. Choudary, A. Dimca, Koszul complexes and hypersurface singularities, Proc. Amer. Math. Soc. 121(1994), 1009-1016. 4

[5] P. Deligne, A. Dimca, Filtrations de Hodge et par l'ordre du pôle pour les hypersurfaces singulières, Ann. Sci. Ecole Norm. Sup. (4) 23(1990), 645-656. 2

[6] A. Dimca, Singularities and Topology of Hypersurfaces, Universitext, Springer-Verlag, 1992. 2.1, 4, 5

[7] A. Dimca, Sheaves in Topology, Universitext, Springer-Verlag, 2004. 2 
[8] A. Dimca, Tate properties, polynomial-count varieties, and monodromy of hyperplane arrangements, Nagoya Math. J. 206 (2012), 75-97.4

[9] A. Dimca, Syzygies of Jacobian ideals and defects of linear systems, Bull. Math. Soc. Sci.Math. Roumanie, Tome 56(104) No. 2, 2013, 191- 203. 5

[10] A. Dimca, Jacobian syzygies, stable reflexive sheaves, and Torelli properties for projective hypersurfaces with isolated singularities, arXiv:1408.2244, to appear in Algebraic Geometry (Compositio Foundation). 4.5

[11] A. Dimca, M. Saito, Some remarks on limit mixed Hodge structure and spectrum, An. Şt. Univ. Ovidius Constanţa 22(2) (2014), 69-78. 2

[12] A. Dimca, M. Saito, Koszul complexes and spectra of projective hypersurfaces with isolated singularities, arXiv:1212.1081. 1, 2, 2, 2, 2, 3, 4.5.

[13] A. Dimca, M. Saito, Generalization of theorems of Griffiths and Steenbrink to hypersurfaces with ordinary double points, arXiv:1403.4563v4. 1

[14] A. Dimca, M. Saito, L. Wotzlaw, A generalization of Griffiths' theorem on rational integrals II, Michigan Math. J. 58(2009), 603-625. 1, 3, 3, 5, 5, 5, 5

[15] A. Dimca, G. Lehrer, Hodge-Deligne equivariant polynomials and monodromy of hyperplane arrangements, in: Configuration Spaces, Geometry, Combinatorics and Topology, Publications of Scuola Normale Superiore, vol. 14 (2012), 231-253.4

[16] A. Dimca, G. Sticlaru, On the syzygies and Alexander polynomials of nodal hypersurfaces, Math. Nachr. 285 (2012), 2120-2128. 1, 1, 4, 4, 4.4, 5

[17] A. Dimca, G. Sticlaru, Koszul complexes and pole order filtrations, Proc. Edinburg. Math. Soc. 58(2015), 333-354.2.1, 4, 4.2, 4.5, 5, 5

[18] A. Dimca, G. Sticlaru, Computing the monodromy and pole order filtration on Milnor fiber cohomology of plane curves, arXiv: 1609.06818. 3.2

[19] D. Eisenbud, M. Green, J. Harris, Cayley Bacharach theorems and conjectures, Bull. Amer. Math. Soc. 33 (1996), 295-324. 5

[20] Ph. Griffiths, On the period of certain rational integrals I, II, Ann. Math. 90(1969), 460-541. 4

[21] M. Mustaţa, M. Popa, Hodge ideals, arXiv:1605.08088 1] 1]

[22] A. Nobile, On families of singular plane projective curves, Ann. Mat. Pura Appl. 138(1984), 341-378. 1, 3

[23] A. Nobile, Surfaces with nodes in projective space, J. Pure Appl. Algebra 42(1986), 275-285. 1, 1,5

[24] C. Peters, J. Steenbrink, Mixed Hodge Structures, Ergeb. der Math. und ihrer Grenz. 3. Folge 52, Springer, 2008. 1

[25] M. Saito, On b-function, spectrum and rational singularity, Math. Ann. 295(1993), 51-74. 2., 3. 3

[26] M. Saito, Multiplier ideals, b-functions, and spectrum of a hypersurface singularity, Compositio Math. 143 (2007), 1050-1068. 2, 2.1, 4

[27] M. Saito, Bernstein-Sato polynomials and graded Milnor algebras for projective hypersurfaces with weighted homogeneous isolated singularities, arXiv:1609.04801.2

[28] F. Severi, Sul massimo numero di nodi di una superficie de dato ordine dello spazio ordinario o di una forma di un iperspazio, Ann. Mat. Pura. Appl. 25 (1946) 1-41. 1

[29] E. Sernesi, Deformations of Algebraic Schemes, Springer Grundlehren b. 334, Springer-Verlag 2006. 1, 3

[30] E. Sernesi, A smoothing criterion for families of curves, preprint February 20093

[31] E. Sernesi, The local cohomology of the Jacobian ring, Documenta Mathematica, 19 (2014), $541-565.4 .6$

[32] J. Steenbrink, Intersection form for quasi-homogeneous singularities, Compositio Math.

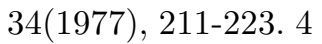


[33] G. Sticlaru, Invariants and rigidity of projective hypersurfaces, Bull. Math. Soc. Sci. Math. Roumanie (N.S.) 58(106) (2015), 103-116.4.6

Université Côte D'Azur, CNRS, LJAD, France

E-mail address: dimca@unice.fr 